\title{
Statistical verification of 2D-to-3D conversion of size and number density of particles
}

\author{
Ahmed Elkholy ${ }^{1}$, Wael Rady ${ }^{2}$, Mahmoud Ahmadein ${ }^{3}$ \\ ${ }^{1}$ Department of Production, Faculty of Industrial Education, Helwan University \\ ${ }^{2}$ Department of curriculum and teaching methods of industrial education, Faculty of Education, Helwan University \\ ${ }^{3}$ Department of Production Engineering and Mechanical Design, Faculty of Engineering, Tanta University
}

\begin{abstract}
Particle/grain size and their number density are commonly characterized in two dimensions (2D) from planar optical or SEM micrographs of polished samples. Accurate conversion of such quantities into the three dimensional (3D) values are necessary for prediction of material properties. Several contradicting conversion correlations are available in literature. The main objective of the current works it to verify some of them. For this purpose, geometrical (3D) models of randomly distributed mono-size spheres were constructed and sliced at different planes. The particle count and their size were variated within the same control volume. The statistical investigations of the date suggested a (2D)-to-(3D) conversion factor of $1.152377 \pm \mathbf{0 . 0 0 9 4 2 7}$, which is very close to some of earlier works [A. N. Sinha, 1999].
\end{abstract}

Keywords: Quantitative metallography, image analysis, grain size, particle size

\section{INTRODUCTION}

Many scientific and applied disciplines (as, for example, materials science and technology, biology and bionics, anatomy and medicine, mineralogy and geology) require a qualitative as well as a quantitative understanding of the properties and the behavior of inorganic and organic materials and their variation during processing, during growth or in use [1]. Quantitative metallography (or, more generally, stereology) deals with the quantitative relationships between measurements made on the twodimensional (2D) plane of polish and the magnitudes of the microstructural features in the three-dimensional (3D) metal or alloy. As material specifications become stricter and performance limits are narrowed, it becomes necessary to specify and control microstructure quantitatively [2].

Metallographic examination constitutes simply a planar section view of a three dimensional structure to determine the size of grains/particles and their number density. The size of grains/particles and their number density are crucial in determining various properties of materials. The grain size has a measurable effect on most of the mechanical properties. For example, at room temperature, the hardness, yield strength, tensile strength, fatigue strength, and impact strength all increase with decreasing grain size of the material. The influence of grain size on the mechanical properties of steel is most commonly expressed in a HallPetch Equation (Eq 1). Similar equations relate the hardness (Eq 2), the cleavage fracture stress of high-strength steels (Eq 3), and the ductile-brittle transition temperature (Eq 4) $[3,4]$. The grain size is also has influence on the hardenability of steels, ductility of brass and in the ductile brittle transition of alloys $[5,6]$.

$$
\begin{aligned}
& \sigma_{y}=\sigma_{0}+K_{y} * d^{-1 / 2} \\
& H_{y}=H_{0}+K_{y} * d^{-1 / 2} \\
& \sigma_{f}=\sigma_{0 f} * K_{f} * d^{-1 / 2} \\
& T_{B}=T_{0}-K_{B} * d^{-1 / 2}
\end{aligned}
$$

Where; $\left(\sigma_{\mathrm{y}}\right)$ : yield stress, $\left(\sigma_{\mathrm{f}}\right)$ : fracture stress, $\left(\sigma_{\mathrm{o}}\right)$ : The friction resistance for dislocation movement within the polycrystalline grains, $(d)$ : The average grain size, $\left(\sigma_{\mathrm{of}}\right)$ and $\left(\mathrm{K}_{\mathrm{f}}\right)$ : the experimentally determined constants, $\left(\mathrm{K}_{\mathrm{B}}\right)$ : Constant in the Hall-Petch relation (temperature dependent) constant, $\left(\mathrm{K}_{\mathrm{y}}\right)$ : Is microstructure-dependent constant.

The grain/particle size measured from (2D) micrographs has to be transformed into (3D) size that is to be used for prediction of the material properties. Measuring the average real size of particles/grains in (3D) is very costly and is replaced by using some empirical correlations to transform the average size in $(2 \mathrm{D}),\left(\bar{D}_{s}\right)$ to a near $(3 \mathrm{D})$ value, $\left(\bar{D}_{\bar{V}}\right)$. However, several transformation correlations that dramatically differ in their results from each other were suggested as given by Eq.5 and $6[6,11]$.

$$
\begin{aligned}
& \bar{D}_{V}=1.16 \bar{D}_{S} \\
& \bar{D}_{V}=1.75 \bar{D}_{S}
\end{aligned}
$$

Three main methods are commonly used to calculate the grain size: the comparison method, the planimetric method and the intercept method. The comparison method may be used if the structure of the material approaches the appearance of one of the standard comparison charts, the intercept and planimetric methods are always applicable for determining an average grain size [7, 12]. There are also several methods have been developed and are being used to obtain the desired information from the section view plane. We may divide them into direct (Serial Sectioning) and indirect methods. In the indirect methods the desired (3D) parameters are deduced from observations of one planar cut. The link between (2D) observations and spatial grain size distributions is provided by specific models based on a series of assumptions. In the direct methods a series of parallel closely spaced metallographic sections observed under the microscope permit the reconstruction of the threedimensional granular arrangement from planar information $[8,11]$. 
Tomographic serial sectioning experiments are conceptually simple, being composed of two steps that are iteratively repeated until completion of the experiment. The first is to prepare a nominally flat surface by e.g., cutting, polishing, ablating, etching, and sputtering, where ideally a constant depth of material removal has occurred between each section. The second step is to collect the 2D characterization data for each section [2, 9, 10, and 13]. In the current study hypothetical samples of randomly dispersed perfect spheres are designed and sliced by using computer programs.

This research aims to study and evaluation of the accuracy of some available relations used in to convert (2D) to (3D) grain size. The effect of different distribution and different sizes of these particles/grains on those relationships are also investigated.

\section{METHODOLOGY}

Hypothetical samples are prepared in a cubic form. They are provided with perfect and randomly dispersed monospheres that resemble the real particles/grains. The perfect spherical shape introduces more accurate estimation of the conversion factor. The random distribution of particles' centers was generated using the MATLAB ${ }^{\circledR}$ software. The overlapping of particles was avoided by maintaining the distance between particle centers $\geqslant$ particle diameter. The 3D sample size, the particle size, and the number density of particles are the input variables to the MATLAB ${ }^{\circledR}$ program. The coordinates of particle centers are the output, which is then imported into the SolidWorks ${ }^{\circledR}$ to generate the randomly dispersed mono-spheres within the cubic model of the samples that have a fixed size of $160 \times 160 \times 160 \mathrm{~mm}^{3}$.

The effect of particle size and their number density on the (2D) -to-(3D) conversion factor are also investigated. For this purpose, two sets of geometrical models of samples with random particle distribution were prepared. One case is shown in Fig.1.

The first set has a constant number grain in the sample 2500 grains, which correspond to particle number density, $\left(\mathrm{N}_{\mathrm{V}}\right)=6.1 \times 10^{-4} \mathrm{~mm}^{-3}$ but with various particle sizes, (Dv), of $8,8.5,9,9.5$ and $10 \mathrm{~mm}$. In the second set, particles have (Dv) $=10 \mathrm{~mm}$, whereas the number of particles varies as; $2350,2400,2450,2500$ and 2550, which corresponds $\mathrm{N}_{\mathrm{V}}$ of; $5.73 \times 10^{-4}, 5.85 \times 10^{-4}, 5.98 \times 10^{-4}, 6.10 \times 10^{-4}$, and $6.22 \times 10^{-4}$ $\mathrm{mm}^{-3}$ respectively.

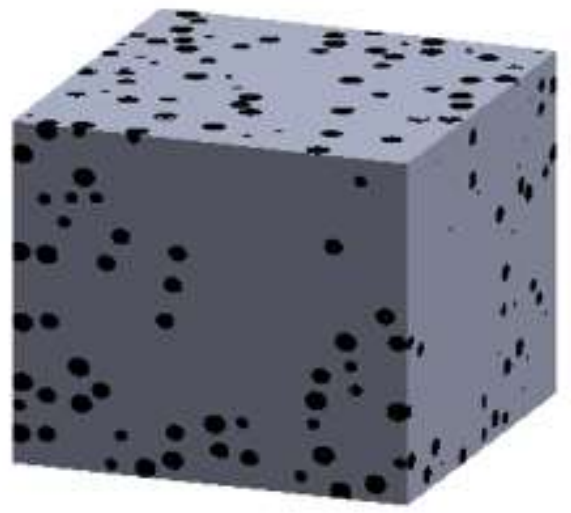

Fig. 1.A 3D construction of the model sample with $10 \mathrm{~mm}$ particle size.

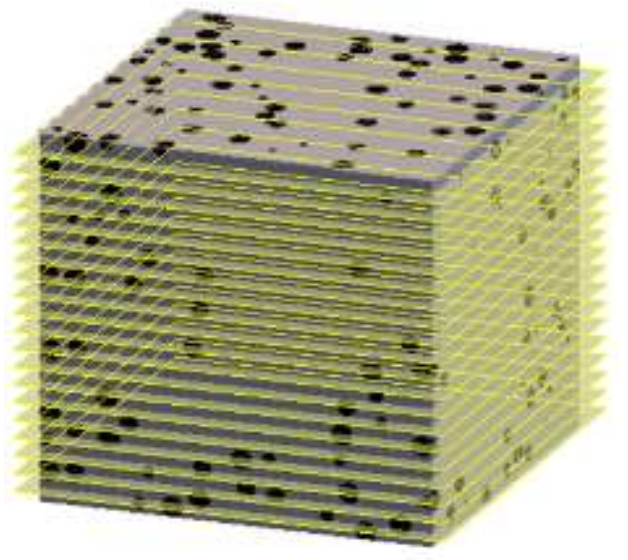

Fig. 2. Sample slicing to produce 2D images for each cut plane.

The 3D model samples were sliced afterwards at different levels with a constant inter-slice spacing as shown in Fig. 2.

Thus, (20) high resolution (2D) images were produced; each of them resembles the real micrographs. Using photoediting software, the produced (2D) images of the resulting slices are transformed into black/white (B/W) images as shown in Fig. 3. The particles have apparently various sizes despite their mono-size in the (3D) sample. This is similar to the case of real micrographs, where grains in the (2D) micrographs appear with various sizes due different prominence of particles/grains with respect to the cut plains. The B/W (2D) images are digitized by the image analyzer. Thus, the average (2D) particle size, the number density of particles and the phase fraction can simply be detected.

The particle/grain count, $\mathrm{N}$, in each plane is calculated as follows: It must be a number of at least 50 grains in the plane section [7], by considering the schematic shown in Fig.3, corner particles are counted as one-fourth and particles intersected by the sides are counted as halves. Accordingly, $\mathrm{N}=(63+0.5 \times 6+2 \times 1 / 4)=66.5$ grains .

The conversion factor $\lambda$ correlates the $2 \mathrm{D}$ average particle size $\left(\bar{D}_{s}\right)$ to the $3 \mathrm{D}$ one as $\left(\bar{D}_{v}=\lambda \times \bar{D}_{s}\right)$. The (3D) size of particles is an input variable as mentioned earlier. The average grain area in the (2D) image ( $\bar{A}$ ) is determined by dividing the total area of grains on the number of grain in

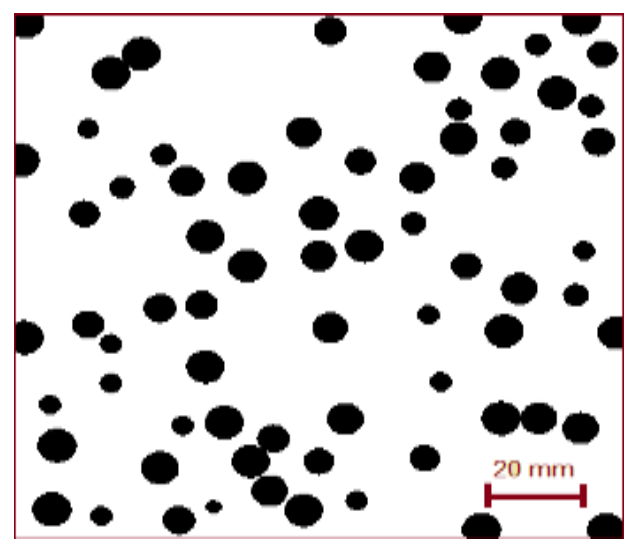

Fig. 3. B/W 2D high resolution image at slices for the analysis purpose. 
the test area. Thus, the average diameter of particle can be given as $\left(D_{s}=2 * \sqrt{ }((A) / \pi)\right)$ and the corresponding $(\lambda)$ can be simply deduced. The process is repeated in each cut plane. Twenty slices are taken for each model sample, which provides a population of 100 data points for each set of parameters. Such data is subjected to further statistical analysis.

\section{ANALYSIS OF RESULTS}

Evaluation of particle/grain size from real micrographs is subjected to several sources of errors. The preparation of samples, operator's competence in microstructure identification, and accuracy of examination equipment and software are of the main sources of errors. However, in the current method the actual (3D) particle size is explicitly known and the average (2D) particle size is accurately determined from the (2D) images by the pixel count. Therefore, the estimated conversion factor is assumed to be of high precision. In particular, the expected sources of variations in estimation of $(\lambda)$ in the current study was considered. Namely, the distribution of particles, the particle size, and their number density. Consequently, a statistical analysis was conducted to evaluate the sensitivity of $(\lambda)$ to such parameters.

As described above, the value of $(\lambda)$ was statistically investigated for two set of geometrical models, the first where the particle diameter variates, and the second set where the number density of particles variates. The impact of variation of $(\mathrm{Dv})$ and $\left(\mathrm{N}_{\mathrm{V}}\right)$ on $\lambda$ is plotted as shown in Fig. 4. It is obvious that the resulting values of $(\lambda)$ have no trend and fluctuates around a certain average of 1.152377 and 1.150142 for the two set of models, respectively.

Due to the random distribution of particle centers, the resulting values of $(\lambda)$ are assumed to obey the Gaussian distribution as shown in Fig.5. Detailed outcome of both sets data is provided in Table 1 . It is obvious that the mean of $(\lambda)$ is very close in both sets.

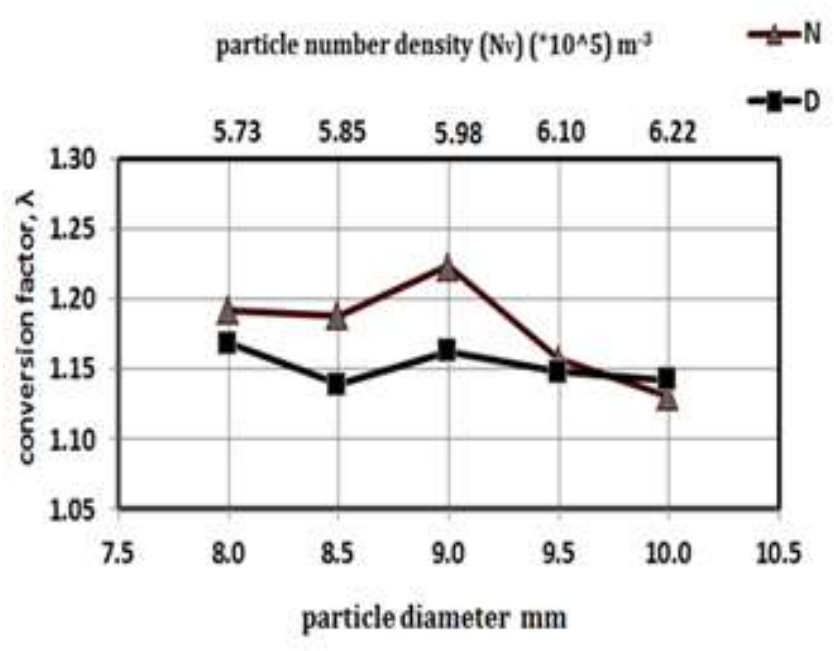

Fig. 4 Calculated $\lambda$ versus the variation of particle diameter (set I) and number of particle in the sample (set II).

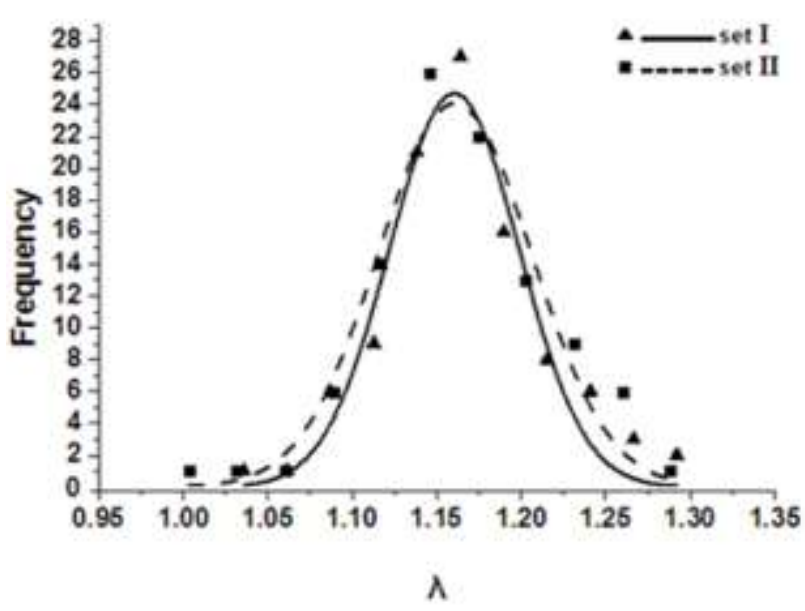

Fig. 5 Normal distribution for the value of the conversion factor $\lambda$

Although the standard deviation $(\sigma)$ is an absolute measure of data dispersion, it is difficult to compare standard deviations when the mean values differ substantially. Thus, it is useful to normalize the standard deviations by dividing $(\sigma)$ by the mean, $(\lambda)$ to get the coefficient of variation $(\mathrm{CV}=(\sigma / \lambda) \times 100)$. It is also useful to calculate the $(95 \%)$ confidence interval. The calculated values are given in Table 1 .

E.g. we can state that the $(\lambda=1.152377 \pm 0.009427)$ with a $(95 \%)$ confidence, based on the results of set I.

From Fig.5, it is clear that the variations of $\left(\mathrm{D}_{\mathrm{V}}\right)$ and $\left(\mathrm{N}_{\mathrm{V}}\right)$ have no significant impact on $(\lambda)$. Also the statistical parameters given in Table 1 for the samples of set I and II are very close. Consequently, it is convenient here to suggest one conversion factor that is calculated as the average of the whole population of set I and II (200 samples). Thus, the current recommends applying $\lambda=1.15126$ as a (2D)-to-(3D) particle size conversion factor.

TABLE I

THE FITTING PARAMETERS OF $\Lambda$ TO THE GAUSSIAN DISTRIBUTION FOR THE SET I AND II DATA VALUES.

\begin{tabular}{|l|l|l|}
\hline \multicolumn{1}{|c|}{ Data set } & \multicolumn{1}{c|}{ I } & \multicolumn{1}{c|}{ II } \\
\hline Mean & 1.152377 & 1.150142 \\
\hline Standard Error & 0.004786 & 0.005127 \\
\hline Median & 1.145459 & 1.146413 \\
\hline Standard Deviation & 0.047857 & 0.051271 \\
\hline Coefficient of variation & 4.152867 & 4.457840 \\
\hline Sample Variance & 0.00229 & 0.002628 \\
\hline Range & 0.256411 & 0.284855 \\
\hline Minimum & 1.035862 & 1.003389 \\
\hline Maximum & 1.292273 & 1.288245 \\
\hline Count & 100 & 100 \\
\hline 95\% Confidence Level & 0.009496 & 0.010173 \\
\hline
\end{tabular}


Vol. $1-2019$

Compared to the previous studies provided in Eq. 4 and 5. The former one is very close to the current study. The conversion factor is supposed to be applicable in case of the microstructures containing particles, precipitates, and during growth of the equiaxed crystals from the melt.

Application of the conversion factor after crystal impingement and in case of space filling equiaxed grains will be subjected to further investigations in the outlook.

\section{CONCLUSIONS:}

The present work describes a simplified method for verification of the conversion of the two dimensional particle/grain size obtained from micrographs into a three dimensional size estimation. It was also aimed to study and assess of the accuracy of some of the contradicting available conversion relationships. In addition, the effect of variation of particle size and its number density on the accuracy of the conversion relationship was investigated. It can be concluded that $(\lambda=1.152377 \pm 0.009427)$ with about $(95 \%)$ confidence. The resulting value is very close to one of the previously suggested correlations. Accordingly, the current obtained conversion factor is recommended to be applied in case of the microstructures containing particles, precipitates, and equiaxed crystals growing from the melt. Application of the conversion factor after crystal impingement and in case of space filling equiaxed grains will be subjected to further investigations in the outlook.
Engineering Research Journal

\section{REFERENCES}

[1] Hans Eckart Exner "Stereology and 3D Microscopy: Useful Alternatives or Competitors in the Quantitative Analysis of Microstructures?" International Society for Stereology \& Image Analysis; 23:73-82, 2004.

[2] E.Ervin Underwood "Metallography and Microstructures" ASM International. Vol 9 187-220, 1992.

[3] Morris Jr., J.W. "The Influence of Grain Size on the Mechanical Properties of Steel" Lawrence Berkeley National Laboratory eScholarship. 2001.

[4] Beata Białobrzeska, Lukasz Konat and Robert Jasinski "The Influence of Austenite Grain Size on the Mechanical Properties of Low-Alloy Steel with Boron" Metals MDPI 7, 26; doi: 10.3390. 2017.

[5] Y.A. Coutinho, S.C.K. Rooney, and E.J. Payton " Analysis of EBSD Grain Size Measurements Using Microstructure Simulations and a Customizable Pattern Matching Library for Grain Perimeter Estimation" ASM International. Vol 48A, 2017.

[6] A. N. Sinha "Image Analysis \& Quantitative Metallography" Scientist National Metallurgical Laboratory Jamshedpur, pp. 135-150, 1999

[7] ASTM "Standard Test Methods for Determining Average Grain Size" ASTM International, 2013.

[8] Michael D. Uchic "Serial Sectioning Methods for Generating 3D Characterization Data of Grain and Precipitate-Scal Microstructures" Springer Science, 31-52, 2011.

[9] Enes Akca, Erwin Trgo "Metallographic Procedures and Analysis - A review" Vol. 3 No. 2, 2015

[10] Donald C. Zipperian, Ph.D "Technologies Metallographic Handbook" PACE Technologies, 66-99, 2011.

[11] K.-P.Huang, Willy "A Simplified Method to Reconstruct a Spatial Grain Distribution from Planar Observations" Prakt. Met. 27, 1990.

[12] George Vander Voort "Introduction to Quantitative Metallography" Buehler Ltd . Volume 1, Issue 5, 1999.

[13] George Vander Voort "Metallography Principles and Practice" ASM International, 2007. 